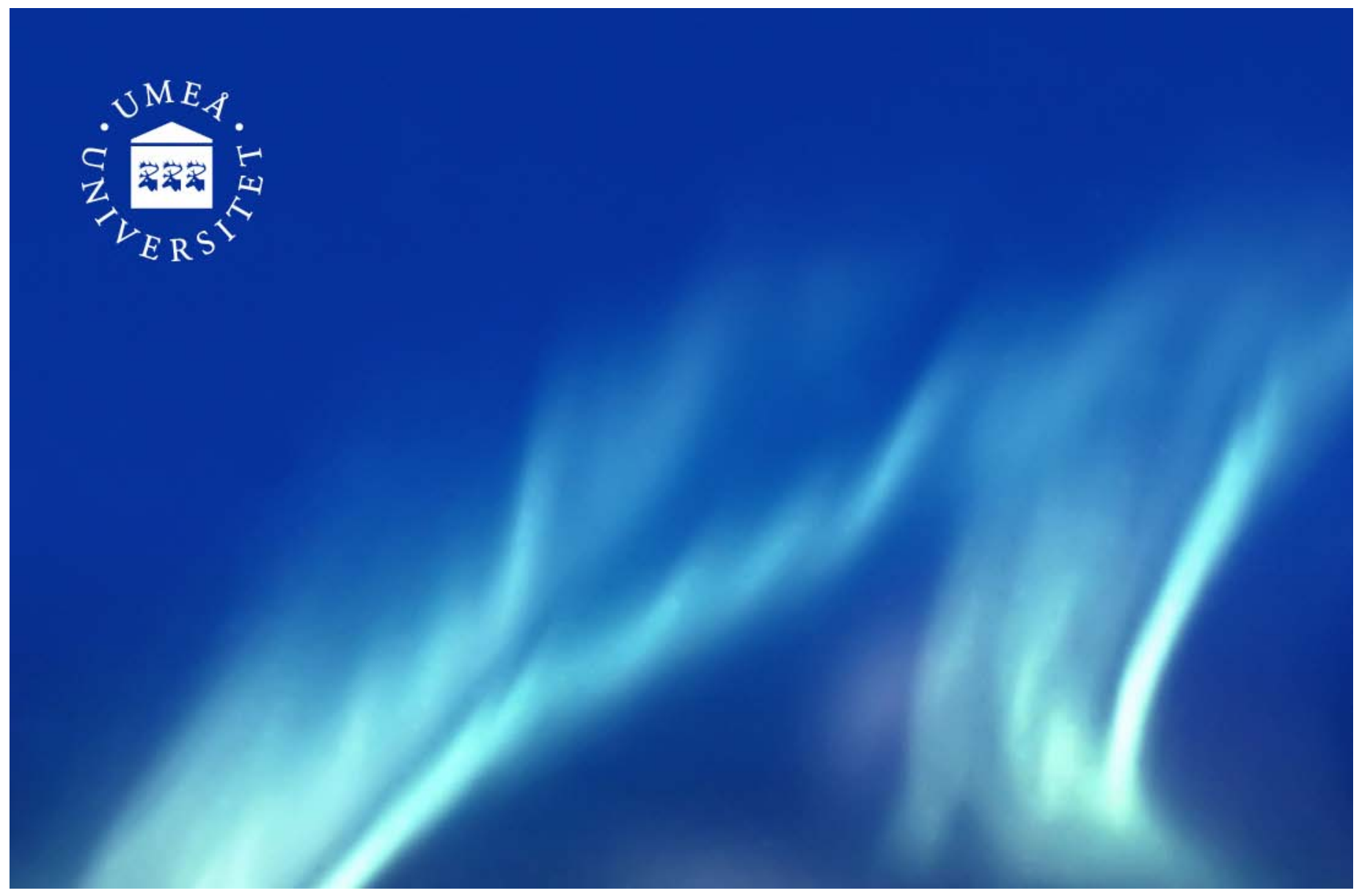

DiVA - Digitala Vetenskapliga Arkivet http://umu.diva-portal.org

This is an author produced version of a paper presented at IEEE Conference on Systems Biology (ISB), Zhuhai, China, September 2-4, 2011.

Citation for the published paper:

Zhao, Jing; Chen, Jie; Yang, Ting-Hong; Holme, Petter

Pathogenesis of Axial Spondyloarthropathy in a Network Perspective

IEEE Conference on Systems Biology (ISB), 2011, p. 41-46

(C) 2011 IEEE. Personal use of this material is permitted. Permission from IEEE must be obtained for all other uses, in any current or future media, including reprinting/republishing this material for advertising or promotional purposes, creating new collective works, for resale or redistribution to servers or lists, or reuse of any copyrighted component of this work in other works. 


\title{
Pathogenesis of axial spondyloarthropathy in a network perspective
}

\author{
Jing Zhao ${ }^{1,2 *}$, Jie Chen ${ }^{3}$, Tinghong Yang ${ }^{1}$, Petter Holme ${ }^{4,5}$ \\ ${ }^{1}$ Department of Mathematics, Logistical Engineering University, Chongqing 400016, China \\ ${ }^{2}$ Department of Natural Medicinal Chemistry, Second Military Medical University, Shanghai, China \\ ${ }^{3}$ Department of Mathematics and Statistics, University of Missouri-Kansas City, MO 641 10-2499, USA \\ ${ }^{4}$ Department of Physics, Umeå University, 90187 Umeå, Sweden \\ ${ }^{5}$ Department of Energy Science, Sungkyunkwan University, Suwon 440-746, Korea \\ *zhaojanne@gmail.com
}

\begin{abstract}
Complex chronic diseases are usually not caused by changes in a single causal gene but by an unbalanced regulating network resulting from the dysfunctions of multiple genes or their products. Therefore, network based systems approach can be helpful for the identification of candidate genes related to complex diseases and their relationships. The Axial spondyloarthropathy $(\mathrm{SpA})$ is a group of chronic inflammatory joint diseases that mainly affects the spine and the sacroiliac joints, yet, the pathogenesis of $\mathrm{SpA}$ remains largely unknown. In this paper, we conducted a networked systems study on the pathogenesis of $\mathrm{SpA}$. We integrated data related to $\mathrm{SpA}$, from the OMIM database, proteomics and microarray experiments of $\mathrm{SpA}$, to prioritize $\mathrm{SpA}$ candidate disease genes in the context of human protein interactome. Based on the top ranked SpA related genes, we constructed a PPI network and identified potential pathways associated with SpA. The PPI network and pathways reflect the well-known knowledge of $\mathrm{SpA}$, i.e., immune mediated inflammation, as well as imbalanced bone modeling caused new bone formation and bone loss. This study may facilitate our understanding of the $\mathrm{SpA}$ pathogenesis from the perspective of network systems.
\end{abstract}

Keywords - spondyloarthropathy, disease gene, protein-protein interaction network, pathway, microarray expression, proteomics

\section{INTRODUCTION}

Axial spondyloarthropathy $(\mathrm{SpA})$ is a family of chronic inflammatory joint diseases of the spine and the sacroiliac joints, and ankylosing spondylitis (AS) is one of the major prototypes of SpA. The two central features of $\mathrm{SpA}$ are inflammation and new bone formation, especially in the spine [1]. Inflammation first occurs around the sites where ligaments attach to bone. As the inflammation heals, there is new bone formation in the ligament, causing the thickening or hardening of the underlying bone, and eventually the fusion of the vertebral bodies and even the spinal stiffness.

It is known that $\mathrm{SpA}$ is associated with multiple genes, such as HLA-B27, TNF and IL23R [2]. However, the pathogenesis of $\mathrm{SpA}$ remains largely unknown. The complexity of the disorder indicates a multifactorial etiology involving multiple biological processes or pathways. In this work, we integrated SpA-associated genes from different resources (known disease genes in the OMIM database, proteomic and microarray experiments) and proposed an approach to prioritize candidate genes in the context of human interactome. We then took out the genes most likely associated with $\mathrm{SpA}$ to construct a protein-protein interaction (PPI) network of SpA and identified potential pathways involved in SpA. The PPI network and pathways could facilitate our understanding of the pathogenesis of SpA.

\section{MATERIALS AND METHODS}

\section{A. Collection of SpA-active genes}

We collected genes active in $\mathrm{SpA}$ from three resources as follows:

(1) the Online Mendelian Inheritance in Man (OMIM) database [3]: we searched the OMIM database with a keyword "Spondyloarthropathy" and found 8 causal genes: HLA-B, TNFA, IL23R, CYP2D6, TNFSF13, TNFSF13B, B2M and COL2A1.

(2) Proteomic experiment results: quantitative proteomics approaches were applied to investigate changes in protein expression in AS (the most common prototype of $\mathrm{SpA}$ ) monocytes in comparison with healthy controls[4]. We used the 43 differentially expressed proteins listed in [4] as active genes in SpA.

(3) The NCBI Gene Expression Omnibus (GEO) database: we searched the GEO database and found 2 microarray experiments related to SpA: GSE1402 (Affymetrix U95Av2) and GSE18781(Affymetrix HG_U133_Plus2). The GSE1402 experiment included a comparison of peripheral blood mononuclear cells (PBMC) from juvenile $\mathrm{SpA}$ with that of normal individuals, and the GSE18781 experiment was an investigation of peripheral blood cells from 18 subjects with SpA and 25 normal individuals. Samples in the GSE18781 experiment were proceeded as two separate sets at different times: $11 \mathrm{SpA}+12$ control subjects in Set 1 and $7 \mathrm{SpA}+13$ control subjects in Set 2 . We treated the results of these two experiments as three separate datasets.

To integrate gene expression data from the two different platforms, we mapped the probe sets of the platforms to 
Entrez Gene ID. This process yielded a set of 9448 genes common to the two platforms. For each gene in a dataset, we calculated the average expression level for probe sets associated to this gene, and filtered out genes whose mean expression ratios (SpA over control) in the two samples are greater than $.67(1 / 1.5)$ and less than 1.5 . In the next step, we converted the expression value to its rank in the common genes [5]. Thereafter, a nonparametric two sample test, the Wilcoxon rank-sum test, was used to test if a gene is differentially expressed in the SpA and control samples and the p-value of the Wilcoxon rank-sum test was obtained. For such a large number of genes being simultaneously tested, the FDR [6] corrected p-values were used for screening differentially expressed genes. Given FDR level of 0.10 , we found genes that are differentially expresses in the two samples for each of the three datasets. We then combined differentially expressed genes in the three datasets and identified 119 distinct genes potentially active in SpA. Finally, the combination of genes from the three recourses yielded a total of 168 SpA-active genes. These 168 potentially $\mathrm{SpA}$-active genes were used in a subsequent analysis (see Section III A).

\section{B. Protein-protein interaction data}

Weighted protein-protein interactions (PPI) of human beings were downloaded from version 8.3 of STRING [7]. STRING includes both physical and functional interactions integrated from numerous sources, including experimental repositories, computational prediction methods and public text collections; uses a scoring system to weigh the evidence of each interaction; and includes the interactions between 14532 proteins (Entrez gene ID) of human genome. We normalized the interaction scores in STRING to the area $[0$, 1] and represented the weighted PPI network as a matrix $\mathbf{W}$.

Innate immunity-relevant human proteins and their interactions were downloaded from the InnateDB database [8] on April 25, 2011. Till the day we downloaded the data, this database includes 2310 human genes and 4819 interactions manually collected by literature review.

\section{Pathway data}

We downloaded pathway data from the FTP service of KEGG [9] (Kyoto Encyclopedia of Genes and Genomes) on April 11, 2011. The KEGG PATHWAY section is a collection of manually drawn pathway maps representing the information on the molecular interaction and reaction networks. The "hsa_pathway.list" file in this section includes a list of known proteins encoded by H. sapiens's genome and the corresponding pathways in which these proteins are involved.

\section{Scoring and ranking genes}

Having a group of SpA-active genes as seeds, we developed a Katz' centrality based index [10] to prioritize genes in the protein-protein interaction network. The starting point from the derivation is the observation that disease genes are typically close, in the associated protein network, to other disease genes [11-13]. Given a weighted human interactome represented as a matrix W corresponding to the interaction strength between proteins, and a set $D$ of $k$ known disease-active genes as seeds, we define vector $\mathbf{x}=\left(x_{1}, x_{2}, \ldots, x_{n}\right)^{T}$ as initially known activity of genes in the disease, with $x_{i}=1$ if gene $i$ is in the set D, $x_{i}=0$ otherwise. Let $\mathbf{s}=\left(s_{1}, s_{2}, \ldots, s_{n}\right)$ be our score vector over the set of genes (where $s_{i}$ indicates how strong $i$ is as a diseasegene candidate),

$$
s_{i}^{t+1}=s_{i}+\phi \sum_{j} w_{i j} s_{j}^{t}
$$

where $t$ indicates iteration time, and $\mathrm{f}$ is a parameter that sets the relative contributions of the activity $\mathbf{x}$ and links in protein interaction networks $\mathbf{W}$ to the score. If $\mathrm{f}$ is small, the known activity is more important; if $\mathrm{f}$ is large, the coupling to the protein neighbors is more important. $f$ needs to be calibrated with real data. The score could be obtained by performing the iteration until the algorithm converges, and then all genes in the protein-protein interaction network could be ranked according to their $s$-scores.

Equation (1) can be represented as matrix form as follows:

$$
\mathbf{s}=\mathbf{x}+\phi \mathbf{W} \mathbf{s}
$$

which can be solved by matrix algebra:

$$
\mathbf{s}=[\mathbf{I}-\phi \mathbf{W}]^{-1} \mathbf{x}
$$

where $[\mathbf{I}-\phi \mathbf{W}]^{-1}$ represents the inverse of the matrix

$\mathbf{I}-\phi \mathbf{W}$. We solved equation (3) by Jacobi iteration algorithm.

The known $k$ disease-active genes were used to determine the parameter $f$. For different values of $f$, we performed leave-one-out cross-validation on the known disease-active gene set $D$. Then $\mathrm{f}$ was taken as the value that resulted in the highest average rank of known disease genes.

\section{E. $k$-core and $k$-core layer}

The $k$-core of a graph is the maximal subgraph such that all its nodes has at least $k$ links within the subgraph [14, 15]. The $k$-core layer $l_{k}$ is defined as the set of nodes that belong to $k$-core but not to $k+1$-core, i.e., $k$-core is the union of $k+1$ core and $k$-core layer. A $k$-core subgraph of a graph can be generated by recursively deleting the vertices from the graph whose present degree is less than $k$. This process can be iterated to gradually zoom into the more connected parts of the network. The higher-level core corresponds to more densely connected part of the network. See Fig. 1 for an explanation. 


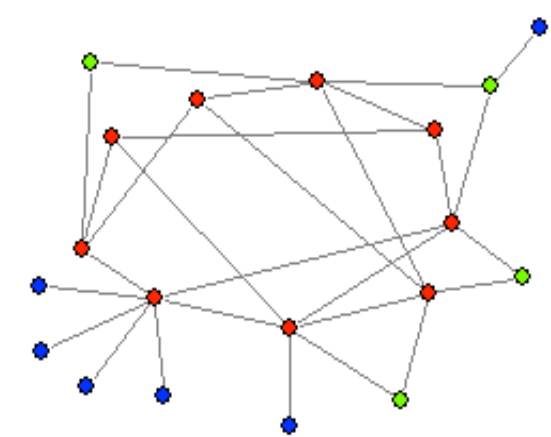

Fig. 1 Illustration of $k$-core and $k$-core layer. The 1-core layer are the blue nodes, the 2-core layer are green. The 1-core is the whole graph, the 2-core is subgraph consisting of green and red nodes and the 3-core is the subgraph consisting of red nodes.

\section{F. P-value}

If we randomly draw $n$ samples from a finite set, the probability of getting $i$ samples with the desired feature by chance obeys the hypergeometric distribution with the following probability mass function:

$$
f(i)=\frac{\left(\begin{array}{c}
K \\
i
\end{array}\right)\left(\begin{array}{c}
N-K \\
n-i
\end{array}\right)}{\left(\begin{array}{c}
N \\
n
\end{array}\right)}
$$

where $N$ is the size of the set, $K$ is the number of items with the desired feature in the set. Then the probability, defined as the P-value, of getting at least $k$ samples with the desired feature by chance can be obtained as the following, using the hypergeometric cumulative distribution function (CDF),

$$
P=1-\sum_{i=0}^{k-1} f(i)=1-\sum_{i=0}^{k-1} \frac{\left(\begin{array}{c}
K \\
i
\end{array}\right)\left(\begin{array}{c}
N-K \\
n-i
\end{array}\right)}{\left(\begin{array}{c}
N \\
n
\end{array}\right)}
$$

Given a significance level a, a P-value smaller than a demonstrates a low probability that the items with the desired feature are chosen by chance. Hence this P-value can be used to measure whether the $n$ samples drawn from the set is more enriched with items of the desired feature than would be expected by chance [16].

\section{RESULTS AND DISCUSSION}

\section{A. Ranking genes in the PPI network}

We integrated OMIM data with the results of the proteomics and microarray experiments and obtained 168 genes potentially active in the axrial spondyloarthropathy disease. A total of 128 of the 168 genes were found present in the STRING PPI network, and these genes were used as seeds to construct the disease gene activity vector $\mathbf{x}$ in equation (3). For all the genes in the PPI network, we calculated the $s$-score vector by equation (3) and ranked the genes accordingly. We first determined the parameter $\mathrm{f}$ by leave-one-out cross-validation. Specifically, applying different values of $\mathrm{f}$ in the area $(0,1 / 100)$, we successively took out one disease active gene and used the left genes as input to rank this one and checked the average ranks of all the seed genes. In this way, $\mathrm{f}$ was taken as $5 / 1000$, which resulted in the highest average rank of seed genes. Then we set $\mathrm{f}$ as 5/1000 and scored all the genes in the human PPI network according to equation (3).

\section{B. PPI network of SpA}

The $s$-score of a gene indicates its possibility associated with the disease. Setting $10 \%$ as a cut-off, we took out the genes whose $s$-scores were top $10 \%$ of all genes in the PPI network. We identified a total of 379 genes. Then we limited interactions in the STRING database to weights of at least 0.5 , which corresponds to a medium-confidence human genome PPI network [7], and constructed a subnetwork spanned by these 379 genes. Finally, we obtained a PPI network associated with SpA, which included 367 nodes and 10906 edges.

Including 10906 interactions with strength at least 0.5 between only 367 proteins, the PPI network of SpA is densely connected. To understand its topology, we conducted $k$-core decomposition on the network. A so-called $k$-core decomposition is a way to visualize both of the connectivity of neighborhoods of nodes and their centrality [15]. In short, a k-core decomposition is obtained by iteratively deleting low-degree nodes to achieve a sequence of $k$-cores (maximal subgraphs with minimal degree $k$, see the Methods section). By and large, following the k-core decomposition is similar to zooming into the more central and more interconnected parts of the network. For the PPI network we studied, the innermost core is the 45-core, which includes 197 nodes. In Fig. 2, we illustrate the node distribution in the hierarchical $k$-core layers of the PPI network and the number of seed genes in the core layers. This figure suggests that the network topology exhibits a core-periphery dichotomy $[17,18]$ - about two thirds of nodes in this network interact frequently and are thus interconnected densely to form an inner 4l-core, while others communicate with fewer nodes and scatter in different outer core layers to form the periphery of the network. Fig. 2 also shows that most seed genes locate at the periphery part whereas the inner core includes most of the non-seed genes. Specifically, a total of 116 seed genes appear in this network, in which only 14 are located in the inner core. All nodes in the core layers $1 \sim 20$ and about half nodes in the core layers $21 \sim 40$ are seed nodes. Therefore, as shown in Fig.3, we partition the network into three parts accordingly - the inner core is the 4l-core, and the medium and outer layers correspond to core layers $21 \sim 40$ and 1 20, respectively. It can be seen that seed genes in the outer and medium layers tend to interact with genes in the inner core, 
indicating that the inner core could be the modulating center of the network.

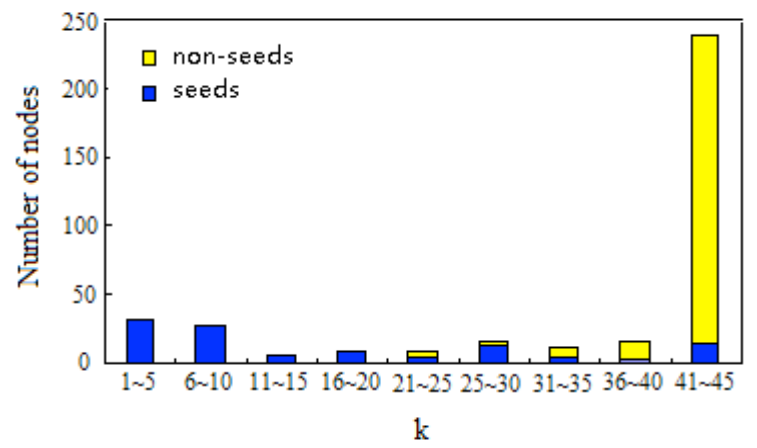

Fig. 2 Distribution of $k$-core layers and seed nodes in the SpA PPI network

Furthermore, we searched the DrugBank database [19] for drugs treating SpA and their protein targets. We mapped the protein targets onto the SpA PPI network and found 6 targets in this network, i.e., PTGS2, MMP2, MAPK3, HRAS, EGF and ALB. They are drug targets for the two main classes of AS drugs [1]: non-steroidal anti-inflammatory drug such as Aspirin, Celecoxib and Sulindac; and diseasemodifying antirheumatic drug such as Thalidomide and Methotrexate. As shown in Fig.3, five of the six targets are located in the inner core of the SpA PPI network, and only ALB situates at the medium layer, suggesting that the drugs may interfere with the disease by acting on proteins in the core.

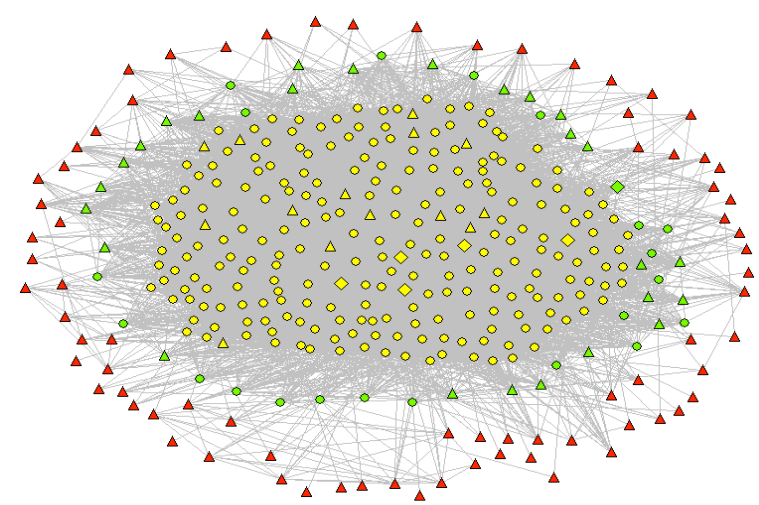

Fig.3 Core-periphery topology of the SpA PPI network. Yellow, green and red nodes represent the inner core, the medium and outer core layer, respectively. Triangles are seed genes, and diamonds are targets of drugs for SpA.

To explore the implications of this PPI network to SpA, we conducted gene ontology (GO) analysis. We used the Pvalue to quantitatively measure whether this PPI network is statistically significantly enriched with genes of a specific Gene ontology (GO) term. In Table I we listed the most significantly enriched GO terms presented in the network, with $p$-values smaller than $10^{-40}$. It can be seen that proteins in this network are significantly involved in biological processes of immune system, such as the regulation of granulocyte macrophage, natural killer cell proliferation, as well as the activation of leukocytes, lymphocytes and $\mathrm{T}$ cells, in consistent with the immune-mediated feature of $\mathrm{SpA}$.

TABLE I

SELECTION OF THE MOST SIGNIFICANTLY ENRICHED GO TERMS IN THE SPA PPI NETWORK

\begin{tabular}{|l|l|l|l|}
\hline GO ID & GO Term & $\begin{array}{l}\text { Total } \\
\text { genes }\end{array}$ & $\begin{array}{l}\text { Mapped } \\
\text { genes }\end{array}$ \\
\hline 0032725 & $\begin{array}{l}\text { positive regulation of granulocyte } \\
\text { macrophage colony-stimulating } \\
\text { factor production }\end{array}$ & 5 & 5 \\
\hline 0032819 & $\begin{array}{l}\text { positive regulation of natural } \\
\text { killer cell proliferation }\end{array}$ & 6 & 6 \\
\hline 0002376 & immune system process & 1429 & 124 \\
\hline 0006950 & response to stress & 2520 & 155 \\
\hline 0051716 & cellular response to stimulus & 3999 & 186 \\
\hline 0010941 & regulation of cell death & 1034 & 99 \\
\hline 0042127 & regulation of cell proliferation & 939 & 92 \\
\hline 0002694 & regulation of leukocyte activation & 288 & 53 \\
\hline 0051249 & $\begin{array}{l}\text { regulation of lymphocyte } \\
\text { activation }\end{array}$ & 261 & 51 \\
\hline 0050863 & regulation of T cell activation & 207 & 46 \\
\hline
\end{tabular}

The immune system process protects human beings against diseases with increasing specificity. The innate immune system provides an immediate, but non-specific response to invading pathogens; and the adaptive immune system, which is activated by the innate response in case that pathogens successfully evade the innate response, adapts its response to improve its recognition of the pathogen. The innate immune response is important in the initiation of and interplay with the adaptive immune response. To investigate the association between the innate immune response and SpA, we constructed a subnetwork of the SpA PPI network involved in the innate immune response, by mapping proteins of the SpA network onto the PPI network of human innate immune response constructed from the InnateDB database (see Fig.4). This subnetwork includes more than half nodes of the SpA network, suggesting that the dysfunction of the innate immune system could be associated with the development of SpA. As can be seen in Fig.4, the innate immune subnetwork is significantly enriched with core nodes and non-seed nodes of the SpA PPI network (p-values are $7.01 \times 10^{-12}$ and $2.14 \times 10^{-7}$, respectively). This observation indicates that a very large fraction of proteins identified by our algorithm (non-seed nodes) are involved in the innate immune response, suggesting that in the human genome PPI network, the known SpA active proteins (seed nodes in our study) are close to a common group of innate immune proteins. Thus our approach reveals the important role of innate immune system in the initiation and development of SpA. 


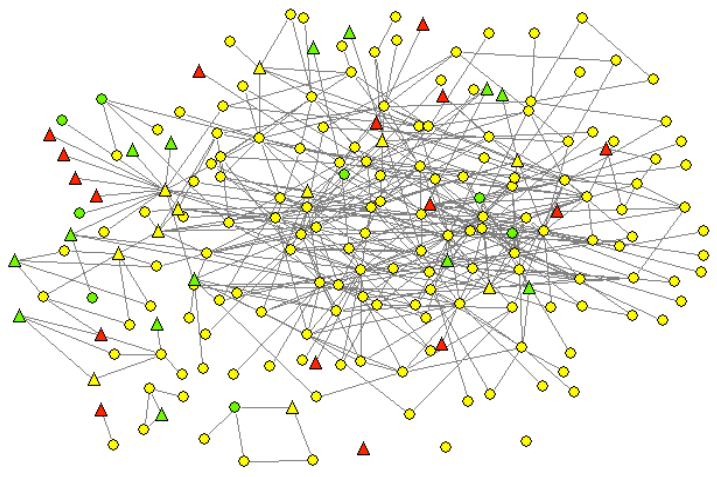

Fig. 4 A subnetwork of the SpA PPI network involved in innate immune response. Yellow, green and red nodes represent the inner core, the medium and outer core layer of the SpA PPI network in Fig.3, respectively. Triangles are seed genes in Fig. 3.

\section{Pathways associated with SpA}

To identify SpA-relevant biological processes, we mapped the $379 \mathrm{SpA}$ associated genes onto the KEGG and Biocarta pathways, respectively. Given significance level $\alpha=0.05$, we found that a total of 27 KEGG pathways are significantly enriched with genes in this group (see Table II). Similar pathways in the Biocarta database are also enriched with SpA associated genes. In Table III we just list four SpA gene enriched pathways included in the Biocarta database but not in the KEGG database.

A central feature of $\mathrm{SpA}$ is inflammation, one of the first responses of the immune system to infection or irritation. As listed in Table II, $\mathrm{SpA}$ is related to a large fraction of pathways in immune system. Some other pathways, although not classified into immune system in the KEGG database, have been known to be highly associated with the function of immune response, such as apoptosis [20], MAPK signaling pathway [21], and cell adhesion molecule interactions [22]. Specifically, Table II includes several pathways related to pathogen recognition and inflammatory signalling in innate immune defences, in which the most important one is the Toll-like receptor (TLR) signalling pathway. The innate immune system relies on pattern recognition receptors (PRRs) to detect distinct pathogenassociated molecular patterns (PAMPs). Upon PAMP recognition, PRRs trigger a number of different signal transduction pathways. The pathways induced by PRRs ultimately result in the expression of a variety of proinflammatory molecules, such as cytokines, chemokines, cell adhesion molecules, and immunoreceptors, which together orchestrate the early host response to infection, mediate the inflammatory response, and also bridge the adaptive immune response [22] together. The family of TLRs is the major class of PRRs [22]. The association of TLR2 and TLR4 with SpA has been reported [23]. It was noticed that three major signaling pathways are responsible for mediating TLR-induced responses including NF-kB, mitogen-activated protein kinases (MAPKs), and IFN regulatory factors (IRFs) [22], while we found that the two pathways, MAPKs and NF-kB, which play central roles in induction of a proinflammatory response, are involved in $\mathrm{SpA}$. Tumor necrosis factor a (TNFa) is an important upstream protein of the NF-kB pathway, which binds to its receptor to recruit TNF receptor death domain (TRADD) and thus activates NF-kB. TNF inhibitors have been proven highly effective for the treatment of SpA [1]. In addition, we also found that $\mathrm{SpA}$ is associated with some proinflammatory molecule involved pathways, such as the chemokine signaling pathway, natural killer cell mediated cytotoxicity, $\mathrm{Fc}$ epsilon RI signaling pathway, and cell adhesion molecules interaction. These pathways indicate the process of innate immune response in the progress of SpA. On the other hand, it is known that $\mathrm{B}$ and $\mathrm{T}$ lymphocytes are responsible for the adaptive immune response [24]. Table II shows the association of $\mathrm{B}$ and $\mathrm{T}$ cell receptor signalling pathways with $\mathrm{SpA}$, implying their function in the adaptive immune response in SpA. In fact, it has been known that both the innate and adaptive immune responses are involved and interdependent with each other in SpA [25].

TABLE II

KEGG PATHWAYS SIGNIFICANTLY ENRICHED WITH SPA-ASSOCIATED GENES.

\begin{tabular}{|c|c|c|c|}
\hline Pathway class & Pathway name & $\begin{array}{l}\text { Total } \\
\text { genes }\end{array}$ & $\begin{array}{l}\text { Mapped } \\
\text { genes }\end{array}$ \\
\hline \multirow{3}{*}{$\begin{array}{l}\text { Cell } \\
\text { Communication }\end{array}$} & Focal adhesion & 206 & 57 \\
\hline & Adherens junction & 80 & 25 \\
\hline & Gap junction & 93 & 18 \\
\hline \multirow{3}{*}{$\begin{array}{l}\text { Cell Growth and } \\
\text { Death }\end{array}$} & Apoptosis & 89 & 30 \\
\hline & p53 signaling pathway & 69 & 16 \\
\hline & Cell cycle & 119 & 20 \\
\hline Cell Motility & $\begin{array}{l}\text { Regulation of actin } \\
\text { cytoskeleton }\end{array}$ & 219 & 45 \\
\hline \multirow{4}{*}{$\begin{array}{l}\text { Endocrine } \\
\text { System }\end{array}$} & Insulin signaling pathway & 138 & 35 \\
\hline & $\begin{array}{l}\text { Adipocytokine signaling } \\
\text { pathway }\end{array}$ & 67 & 20 \\
\hline & GnRH signaling pathway & 108 & 23 \\
\hline & Melanogenesis & 105 & 16 \\
\hline \multirow[t]{8}{*}{ Immune System } & Chemokine signaling pathway & 198 & 60 \\
\hline & $\begin{array}{l}\text { T cell receptor signaling } \\
\text { pathway }\end{array}$ & 110 & 47 \\
\hline & $\begin{array}{l}\text { B cell receptor signaling } \\
\text { pathway }\end{array}$ & 75 & 31 \\
\hline & $\begin{array}{l}\text { Leukocyte transendothelial } \\
\text { migration }\end{array}$ & 122 & 37 \\
\hline & $\begin{array}{l}\text { Fc epsilon RI signaling } \\
\text { pathway }\end{array}$ & 79 & 34 \\
\hline & $\begin{array}{l}\text { Natural killer cell mediated } \\
\text { cytotoxicity }\end{array}$ & 138 & 43 \\
\hline & $\begin{array}{l}\text { Toll-like receptor signaling } \\
\text { pathway }\end{array}$ & 102 & 44 \\
\hline & Hematopoietic cell lineage & 88 & 33 \\
\hline \multirow{6}{*}{$\begin{array}{l}\text { Signal } \\
\text { Transduction }\end{array}$} & Jak-STAT signaling pathway & 155 & 51 \\
\hline & VEGF signaling pathway & 77 & 27 \\
\hline & ErbB signaling pathway & 87 & 37 \\
\hline & TGF-beta signaling pathway & 87 & 18 \\
\hline & MAPK signaling pathway & 276 & 57 \\
\hline & Wnt signaling pathway & 152 & 18 \\
\hline $\begin{array}{l}\text { Signaling } \\
\text { Molecules and }\end{array}$ & $\begin{array}{l}\text { Cell adhesion molecules } \\
\text { (CAMs) }\end{array}$ & 136 & 20 \\
\hline
\end{tabular}


Another prominent feature of $\mathrm{SpA}$ is new bone formation; meanwhile bone loss is also a common finding in SpA [26]. As can be seen in Table III, SpA is associated with bone remodeling, a process that maintains bone density and structure through a balance of bone resorption by osteoclasts and bone deposition by osteoblasts. Both ossification and osteoporosis symptoms of $\mathrm{SpA}$ are consequences of an imbalance in the regulation of these two sub-processes of bone remodelling. It is known that the WNT pathway regulates the balance between osteoclast and osteoblast function [27], verifying our result that $\mathrm{SpA}$ is associated with the WNT pathway (see Table II).

TABLE III

SELECTION OF BIOCARTA PATHWAYS SIGNIFICANTLY ENRICHED WITH SPAASSOCIATED GENES

\begin{tabular}{|l|l|l|}
\hline Pathway name & $\begin{array}{l}\text { Total } \\
\text { genes }\end{array}$ & $\begin{array}{l}\text { Mapped } \\
\text { genes }\end{array}$ \\
\hline Bone Remodelling & 13 & 6 \\
\hline Cytokines and Inflammatory Response & 26 & 17 \\
\hline NF-kB Signaling Pathway & 22 & 13 \\
\hline TNFR2 Signaling Pathway & 17 & 8 \\
\hline
\end{tabular}

\section{CONCLUSIONS}

We have extracted data related to $\mathrm{SpA}-$ known SpA causal genes from the OMIM database, proteomic experiments from literature, and microarray experiments from the GEO database, and subsequently used these genes as seeds to rank genes in the human PPI network by an $s$ score measuring their proximity to the seeds in the PPI network. We considered 379 top ranked genes as associated with $\mathrm{SpA}$ with high possibility. Based on these genes, we

[1] J. Braun and J. Sieper, "Ankylosing spondylitis," Lancet vol. 369, pp. 1379-90, 2007.

[2] T. Pham, "Pathophysiology of ankylosing spondylitis: What's new?," Joint Bone Spine, vol. 75, pp. 656-660, 2008.

[3] A. Hamosh, A. F. Scott, J. S. Amberger, C. A. Bocchini, and V. A. McKusick, "Online Mendelian Inheritance in Man (OMIM), a knowledgebase of human genes and genetic disorders," Nucleic Acids Res vol. 33, pp. D514-517, 2005.

[4] C. Wright, M. Edelmann, K. diGleria, S. Kollnberger, H. Kramer, S. McGowan, K. McHugh, S. Taylor, B. Kessler, and P. Bowness, "Ankylosing spondylitis monocytes show upregulation of proteins involved in inflammation and the ubiquitin proteasome pathway," Annals of the Rheumatic Diseases, vol. 68, pp. 1626-1632, 2009.

[5] H.-C. Liu, C.-Y. Chen, Y.-T. Liu, C.-B. Chu, D.-C. Liang, L.-Y. Shih, and C.-J. Lin, "Cross-generation and cross-laboratory predictions of Affymetrix microarrays by rank-based methods," Journal of Biomedical Informatics, vol. 41, pp. 570-579, 2008

[6] Y. Benjamini and Y. Hochberg, "Controlling the False Discovery Rate: A Practical and Powerful Approach to Multiple Testing," Journal of the Royal Statistical Society. Series B (Methodological), vol. 57, pp. 289-300, 1995 .

[7] L. J. Jensen, M. Kuhn, M. Stark, S. Chaffron, C. Creevey, J. Muller, T. Doerks, P. Julien, A. Roth, M. Simonovic, P. Bork, and C. von Mering, "STRING 8- a global view on proteins and their functional

constructed a PPI network and identified potential pathways associated with SpA. The PPI network exhibits a coreperiphery topology, in which most seed genes are located at the periphery part, while the inner core aggregates the nonseed genes enriched with innate immune genes and drug targets for SpA, suggesting that the core could be the modulating center of the network. The pathways represent the well-known knowledge of SpA, i.e., immune mediated inflammation, as well as imbalanced bone modeling caused new bone formation and bone loss. This network approach represents an alternative method for analyzing the complex effects of candidate genes related to complex diseases. It may shed new lights on the pathogenesis of axial spondyloarthropathy from the perspective of network systems.

\section{ACKNOWLEDGMENT}

This research was supported by the National Natural Science Foundation of China (10971227); the Special Program for New Drug Innovation of the Ministry of Science and Technology, China (2009ZX09311-001, 2008ZX09101-Z-029); the Swedish Foundation for Strategic Research, the Swedish Research Council and the WCU program through NRF Korea funded by MEST R31-200810029-0.

\section{REFERENCES}

interactions in 630 organisms," Nucleic Acids Research, vol. 37, pp. D412D416, 2009

[8] D. J. Lynn, G. L. Winsor, C. Chan, N. Richard, M. R. Laird, A Barsky, J. L. Gardy, F. M. Roche, T. H. W. Chan, N. Shah, R. Lo, M. Naseer, J. Que, M. Yau, M. Acab, D. Tulpan, M. D. Whiteside, A Chikatamarla, B. Mah, T. Munzner, K. Hokamp, R. E. W. Hancock, and F. S. L. Brinkman, "InnateDB: facilitating systems-level analyses of the mammalian innate immune response," Mol Syst Biol, vol. 4, 2008.

[9] M. Kanehisa and S. Goto, "KEGG: Kyoto Encyclopedia of Genes and Genomes," Nucleic Acids Res., vol. 28, pp. 27-30, 2000.

[10] L. Katz, "A new status index derived from sociometric analysis," Psychometrika, vol. 18, pp. 39-43, 1953.

[11] K.-I. Goh, M. E. Cusick, D. Valle, B. Childs, M. Vidal, and A.-L. Barabási, "The human disease network," Proc Natl Acad Sci USA, vol. 104, pp. 8685-8690, 2007.

[12] J. Zhao, P. Jiang, and W. Zhang, "Molecular networks for the study of TCM pharmacology," Briefings in Bioinformatics vol. 11, pp. 417430, 2010 .

[13] F. Barrenas, S. Chavali, P. Holme, R. Mobini, and M. Benson, " Network properties of complex human disease genes identified through genome-wide association studies," PLoS ONE, vol. 4, pp. e8090, 2009.

[14] S. B. Seidman, "Network structure and minimum degree," Social Networks, vol. 5, pp. 269-287, 1983.

[15] S. Wuchty and E. Almaas, "Peeling the yeast protein network," Proteomics, vol. 5, pp. 444-449, 2005. 
[16] S. Tavazoie, J. D. Hughes, M. J. Campbell, R. J. Cho, and G. M. Church, "Systematic determination of genetic network architecture," Nat Genet, vol. 22, pp. 281-285, 1999.

[17] P. Holme, "Core-periphery organization of complex networks," Phys. Rev. E, vol. 72, pp. 046111, 2005.

[18] J. Zhao, G.-H. Ding, L. Tao, H. Yu, Z.-H. Yu, J.-H. Luo, Z.-W

Cao, and Y.X. Li, "Modular co-evolution of metabolic networks," BMC Bioinformatics, vol. 8, pp. 311, 2007.

[19] D. S. Wishart, C. Knox, A. C. Guo, S. Shrivastava, M. Hassanali, P. Stothard, Z. Chang, and J. Woolsey, "DrugBank: a comprehensive resource for in silico drug discovery and exploration," Nucleic Acids Res, vol. 34, pp. D668-672, 2006

[20] T. Mavadas and X. Cullere, "Neutrophil beta2 integrins: moderators of life or death decisions," Trends Immunol, vol. 26, pp. 388-395, 2005.

[21] M. S. Lee and Y.-J. Kim, "Signaling Pathways Downstream of Pattern-Recognition Receptors and Their Cross Talk," Annu. Rev. Biochem., vol. 76, pp. 447-80, 2007.

[22] T. H. Mogensen, "Pathogen Recognition and Inflammatory Signaling in Innate Immune Defenses," Clinical Microbiology Reviews, vol. 22, pp. 240-273, 2009

[23] R. D. Inman and H. S. El-Gabalawy, "The immunology of ankylosing spondylitis and rheumatoid arthritis: a tale of similarities and dissimilarities," Clin Exp Rheumatol, vol. 27 (Suppl. 55), pp. S26-S32, 2009.

[24] A. Bauch and G. Superti-Furga, "Charting protein complexes, signaling pathways, and networks in the immune system," Immunological Reviews, vol. 210, pp. 187-207, 2006

[25] O. FitzGerald and I. McInnes, "Spondyloarthropathy: disease at the crossroads of immunity," Best Practice \& Research Clinical Rheumatology, vol. 20, pp. 949-967, 2006

[26] A. Anandarajah and E. Schwarz, "Bone Loss in the Spondyloarthropathies: Role of Osteoclast, RANKL, RANK and OPG in the Spondyloarthropathies," Adv Exp Med Biol, vol. 649, pp. 85-99, 2009.

[27] S. Goldring and M. Goldring, "Eating bone or adding it: the Wnt pathway decides," Nat Med, vol. 13, pp. 133-134, 2007. 